\title{
GCU
}

Glasgow Caledonian

University

University for the Common Good

\section{Quantification of computational fluid dynamics simulation assists the evaluation of protection by Gypenosides in a zebrafish pain model}

Zhao, Zhenkai ; Xiao, Qing ; Tchivelekete, Gabriel Mbuta; Reilly, James; Jiang, Huirong; Shu, Xinhua

Published in:

Physiology and Behavior

DOI:

10.1016/j.physbeh.2020.113223

Publication date:

2021

Document Version

Author accepted manuscript

Link to publication in ResearchOnline

Citation for published version (Harvard):

Zhao, Z, Xiao, Q, Tchivelekete, GM, Reilly, J, Jiang, H \& Shu, X 2021, 'Quantification of computational fluid dynamics simulation assists the evaluation of protection by Gypenosides in a zebrafish pain model', Physiology and Behavior, vol. 229, 113223. https://doi.org/10.1016/j.physbeh.2020.113223

\section{General rights}

Copyright and moral rights for the publications made accessible in the public portal are retained by the authors and/or other copyright owners and it is a condition of accessing publications that users recognise and abide by the legal requirements associated with these rights.

Take down policy

If you believe that this document breaches copyright please view our takedown policy at https://edshare.gcu.ac.uk/id/eprint/5179 for details

of how to contact us. 
1 Quantification of computational fluid dynamics simulation assists the evaluation

2 of protection by Gypenosides in a zebrafish pain model

3

4 Zhenkai Zhao ${ }^{1}$, Qing Xiao ${ }^{1 \#, ~ G a b r i e l ~ M b u t a ~ T c h i v e l e k e t e ~}{ }^{2}$, James Reilly², Huirong

$5 \quad$ Jiang $^{3}$, Xinhua Shu ${ }^{2,4,5 \#}$

61 Department of Naval Architecture, Ocean, and Marine Engineering, University of

7 Strathclyde, Glasgow G4 0LZ, UK

82 Department of Biological and Biomedical Sciences, Glasgow Caledonian University,

9 Glasgow G4 0BA, United Kingdom

3 Strathclyde Institute of Pharmacy and Biomedical Sciences, Glasgow G4 0RE,

United Kingdom

4 Department of Vision Science, Glasgow Caledonian University, Glasgow G4 0BA, United Kingdom

5 School of Basic Medical Sciences, Shaoyang University, Shaoyang, Hunan 422000, P. R. China

\#corresponding author Qing Xiao, qing.xiao@strath.ac.uk; Xinhua Shu,

Xinhua.Shu@gcu.ac.uk

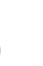

20




\section{Abstract}

In recent years, due to its rapid reproduction rate and the similarity of its genetic structure to that of human, the zebrafish has been widely used as a pain model to study chemical influences on behavior. Swimming behaviors are mediated by motoneurons in the spinal cord that drive muscle contractions, therefore a knowledge of internal muscle mechanics can assist the understanding of the effects of drugs on swimming activity. To demonstrate that the technique used in our study can supplement biological observations by quantifying the contribution of muscle effects to altered swimming behaviours, we have evaluated the pain/damage caused by $0.1 \%$ acetic acid to the muscle of 5 dpf zebrafish larvae and the effect of protection from this pain/damage with the saponin Gypenosides (GYP) extracted from Gynostemma pentaphyllum. We have quantified the parameters related to muscle such as muscle power and the resultant hydrodynamic force, proving that GYP could alleviate the detrimental effect of acetic acid on zebrafish larvae, in the form of alleviation from swimming debility, and that the muscle status could be quantified to represent the degree of muscle damage due to the acetic acid and the recovery due to GYP. We have also linked the behavioral changes to alteration of antioxidant and inflammation gene expression. The above results provide novel insights into the reasons for pain-related behavioral changes in fish larvae, especially from an internal muscle perspective, and have quantified these changes to help understand the protection of swimming behaviors and internal muscle by GYP from acetic acid-induced damage.

\section{Keywords}

Computational fluid dynamics simulation; hydrodynamic performance; gypenosides; acetic acid; zebrafish

(1)

52




\section{Introduction}

The zebrafish is widely recognized as a useful model for scientific and medical research, especially for pain-related study. In its embryonic and larval stage, the zebrafish body is practically transparent, which conveniently allows observation of organ development [1]. Its rapid reproduction rate and cheaper cost, compared to other fish species and rodent animals, afford it a unique and important role in medical research of a wide range of issues [2]. In addition, zebrafish are less sentient, which complies with the 3R criteria [3]. Given that zebrafish share over $70 \%$ of genes with humans [4], studying the effects of pain/damage on zebrafish can provide insight to the human response. Nociception, the sensory system used to detect pain resulting from potential harmful stimuli, is central to the study of body injury [5], with pain being regarded as one of the manifestations of inflammation [6]. Recent studies have shown that both adult and larval zebrafish have a peripheral and central nociceptive processing system that is comparable to that of mammals, including multiple types of nociceptors [7]. However, compared to other animals used in nociception studies, the unique features of the zebrafish allow for the use of distinctive methodologies, including the identification of neurosensory structures in vivo by activation of fluorescent proteins and by observation of characteristic behavioural responses [8, 9]. Together with the previously mentioned advantages, this has allowed the zebrafish to be used as a qualified pain model to study nociception responses. Depending on the types and doses of noxious stimulus applied, the resultant nociceptive responses can be assessed by observing the effect on basic swimming kinematics such as distance travelled and swimming velocity [10, 11]. In order to quantify swimming velocity and fluid effects, particle image velocimetry (PIV) has been used to supplement biological observations [12].

The zebrafish pain model is well established and therefore can be used to study the effect of drugs on pain alleviation and post-pain recovery. Recovery (as indicated by heart rate changes and increased vitality) after exposure to noxious stimuli such as temperature and chemicals, has been observed in zebrafish treated with known analgesics such as ethanol and morphine [13-17]. Behavioural 
improvements following analgesia treatments have also been assessed in terms of time spent active, body curvature alteration, and average velocity over a period of time $[18,19]$.

Most of the above-mentioned biological studies using the zebrafish pain model have focused primarily on the kinematic and hydrodynamic performance caused by the surrounding fluid, but have disregarded the effect of internal muscle mechanics [7, $9,20]$. Locomotion of zebrafish larvae is powered by an axial muscle system driven by motoneurons in the spinal cord [21]. Swimming kinematics are influenced both by internal body mechanics and fluid mechanics [22, 23]. Musculature along the fish body contracts and a bending wave is generated that pushes against and changes the fluid dynamics of the surrounding water, i.e., hydrodynamic force. In turn, this altered hydrodynamic force has an impact on body curvature variations [24]. Under these circumstances, the coupled mechanical interaction determines the motion of the fish through the water. Clearly, internal body mechanics are of tremendous significance in the study of body motion.

In our previous study, inspired by earlier work involving Computational Fluid Dynamics (CFD) simulation of fish swimming [11, 25-28], we built a zebrafish larva pain model to quantify the effects on fish locomotion of different types of drugs, proving that our methodology can quantify the effects of various types of drug influences on zebrafish swimming activity [29]. To extend our research, we have evaluated the protective effects of Gypenosides (GYP) against pain-induced detriment of zebrafish swimming performance. Gypenosides (GYP), a saponin extracted from Gynostemma pentaphyllum, have for centuries been widely used by human beings [30]. In the present day, GYP is approved by the Central Drug Administration of China for use as an over-the-counter medicine in clinics [31]. GYP has been shown to have a range of effects, including antioxidation, antilipidemia, neuroprotection and inflammation reduction [32]. Previous studies have shown that GYP protects against oxidative stress in retinal pigment epithelium cells [33] and vascular endothelial cells [34]. In addition, it has been proved to lower triglyceride, cholesterol and nitrite in acute hyperlipidaemia of rats [35]. It has also been shown to have anti-inflammatory 
effects on aortic lesions of rats and human osteoarthritis chondrocytes [36]. However, protection of GYP against pain in zebrafish has rarely been investigated.

Acid, which can be regarded as a potentially noxious irritant agent that is known to stimulate nociceptors in mammals, has been extensively used in studies involving a number of non-mammalian animals such as fish [9, 14, 37, 38]. Zebrafish larvae have been considered as an appropriate substitute for adult zebrafish [9, 38]. In order to demonstrate that exposure of zebrafish to acid triggers nociception pathways rather than merely inducing stress or anxiety, scientists have studied the levels of cyclooxygenase-2 (cox-2, a gene used as a marker for the activation of nociception pathways) in zebrafish larvae and have found that exposure to a low-concentration acetic acid environment produces behavioral changes that are accompanied by changes in levels of cox-2 [39]. It seems reasonable, therefore, to conclude that the acid-induced behavioral changes can be attributed to nociceptor activation. Studies on nociception in zebrafish larvae have used both acetic acid and citric acid at different concentrations, and have provided evidence that nociceptors in zebrafish larvae respond to low $\mathrm{pH}$ acetic acid $[17,18,40]$.

We have quantified the effect of altered muscle mechanics on a variety of swimming behaviors resulting from treatment of $5 \mathrm{dpf}$ (days post-fertilization) zebrafish larvae. Given that acetic acid at high concentration is known to be harmful to zebrafish larvae [40, 41], we chose to investigate the protective effects of $5 \mu \mathrm{g} / \mathrm{ml}$ GYP on $0.1 \%$ acetic acid, which has been considered to be a threshold concentration for behavioral change [41], to observe and record any alleviation of muscle inflammation following treatment with GYP, which have been shown to have anti-inflammatory effects [42, 43]. The varied swimming behaviors among the tested groups were correlated with the altered muscle forces and power. Quantification of the internal muscle mechanics was made and compared with the biological data from real-time PCR to establish the extent of the protective effects by GYP against inflammation caused by high concentration of acetic acid.

\section{Materials and Methods}


Animal work was carried out in compliance with the Animal Ethics and

Welfare Committee, Department of Biological and Biomedical Sciences, Glasgow Caledonian University, and UK Home Office under Project License PPL 60/4169.

2.2. Experimental setup

The experiment used a high-speed camera (CSI5003XE, Circuit Specialists, USA) to record the locomotion of 5 dpf zebrafish larvae (shown in Fig 1A). The capture of zebrafish larva by the high-speed camera is shown in Fig. 1B. Although the tail beat frequency of 5 dpf zebrafish larvae can reach up to $85 \mathrm{~Hz}$ [44], the fish we used had a tail beat frequency of less than $70 \mathrm{~Hz}$, which means that 7-8 frames within one tail beat cycle was sufficient to capture the fish tail motion and extract motion equations; therefore, the frame rate per second (fps) of the camera was set at $500 \mathrm{fps}$ to capture the locomotion of zebrafish larvae accurately. Prior to the experiment, determination of appropriate acetic acid concentration was carried out. We found that when exposed to $0.2 \%$ acetic acid the zebrafish larvae remained motionless most of the time, and so little or no locomotor behavior could be observed. The threshold of acetic acid concentration for zebrafish behavioral changes was established previously as $0.1 \%$ [41], and so this concentration was selected for the current experiment. Initial toxicity assessment of GYP was performed by treating zebrafish at $4 \mathrm{dpf}$ for 24 hours with GYP at 0, 2.5, 5.0, 15 and $25 \mu \mathrm{g} / \mathrm{ml}$. 2.5 and $5.0 \mathrm{ug} / \mathrm{ml}$ showed no toxicity; 15 and $25 \mu \mathrm{g} / \mathrm{ml}$ showed toxic effects as indicated by reduced heart rate (data not shown). Consequently, $5 \mu \mathrm{g} / \mathrm{ml}$ GYP was chosen for the current experiment. Eighty 4-dpf zebrafish larvae were divided into four groups and each group contained 20 fish larvae. Group 1 and Group 2 were treated with $5 \mu \mathrm{g} / \mathrm{ml}$ GYP in E3 medium (5mM $\mathrm{NaCl}, 0.17 \mathrm{mM} \mathrm{KCl}, 0.33 \mathrm{mM} \mathrm{CaCl} 2,0.22 \mathrm{mM} \mathrm{MgSO}_{4}$, and 0.1\% methylene blue); Group 3 and Group 4 were kept in E3 medium. The zebrafish larvae were kept in a homothermic incubator at $27^{\circ} \mathrm{C}$ for 24 hours. At $5 \mathrm{dpf}$, Group 2 and Group 4 remained untreated as control groups. At this time, their swimming behaviors were recorded for ten minutes to assess the pre-stimulation behavior. For Group 1 and Group 3, 0.1\% acetic acid was added to the petri dishes and the swimming behaviors were immediately recorded for a period of ten minutes. For all groups, fish larvae were able 
175 to swim freely with no environmental impediments while being observed

continuously by the camera. Swimming behaviors of all the fish larvae were post-processed with in-house MATLAB code to be prepared as input for the CFD simulation. The original image recorded from the camera was converted to a binary image consisting of the sketch of zebrafish larva only with "im2bw" function in $M A T L A B$ image processing toolbox. With some adjustments and "bwboundaries" function in $M A T L A B$, a binary image of zebrafish can be extracted, and the entire position vector can be obtained for points distributed on fish outline. All images were skeletonized into a single backbone curve using functions "bwmorph" and "thin" operation. In order to estimate body curvatures, the backbone was divided into several segments and these segments were simplified as connected straight lines to calculate relative orientation variation with time between two adjacent segments using $M A T L A B$ curve fitting toolbox (shown in Fig 1C and Fig 1D). Cruising, during which the swimming movements are repeated cyclically [45], is known to be essential for fish larvae in order to allow coverage of sufficient distance for migration and dispersal [46]. In this experiment, only cruising period was considered, including swimming cyclically. The fitted relative orientation equations between each two body segments are expressed with $\operatorname{asin}(\omega t)+b \cos (\omega t)$, where $\omega$ is the swimming frequency. During cyclic swimming, the travelling wave of curvature travels along the fish body at a near constant rate [47], thus an averaged frequency has been selected for the entire relative orientation.

\subsection{Quantitative real-time polymerase chain reaction ( $q R T-P C R$ )}

Total RNA was isolated from untreated and treated zebrafish embryos using Trizol Reagent (Sigma, UK) according to the manufacturer's guidance. The complementary deoxyribonucleic acid (cDNA) was synthesized using a High-Capacity cDNA Reverse Transcription Kit (Applied Biosystems, UK). Gene expression was measured by qRT-PCR assay using a Platinum ${ }^{\circledR}$ SYBR ${ }^{\circledR}$ Green PCR kit (Thermo Fisher Scientific, UK). Relative expression of target gene was determined by normalization to the expression of the housekeeping gene ( $\beta$-actin) in the untreated 
and treated samples, using $2^{\wedge}-\Delta \Delta C T$ formula. The primer sequences of the genes used 205 are listed in Table 1.

206

Table 1 Primers used for qRT-PCR

\begin{tabular}{|l|l|l|l|l|}
\hline Genes & Forward primers 5'-3' & Reverse primers 5'-3' & TM$^{\circ} \mathrm{C}$ & $\begin{array}{l}\text { PCR product } \\
\text { (bp) }\end{array}$ \\
\hline $\begin{array}{l}\beta-A C T I \\
\mathrm{~N}\end{array}$ & $\begin{array}{l}\text { ACTGTATTGTCTGGTG } \\
\text { GTAC }\end{array}$ & $\begin{array}{l}\text { ATCTCCTGCTTGCTAAT } \\
\text { CC }\end{array}$ & 69.7 & 198 \\
\hline IL-1 $\beta$ & $\begin{array}{l}\text { TTCCCCAAGTGCTGCT } \\
\text { TATT }\end{array}$ & $\begin{array}{l}\text { AAGTTAAAACCGCTGT } \\
\text { GGTCA }\end{array}$ & 54.6 & 149 \\
\hline IL-6 & $\begin{array}{l}\text { TCAACTTCTCCAGCGT } \\
\text { GATG }\end{array}$ & $\begin{array}{l}\text { TCTTTCCCTCTTTTCCTC } \\
\text { CTG }\end{array}$ & 55.1 & 75 \\
\hline TNF- $\alpha$ & $\begin{array}{l}\text { ACCAGGCCTTTTCTTC } \\
\text { AGGT }\end{array}$ & $\begin{array}{l}\text { GCATGGCTCATAAGCA } \\
\text { CTTGTT }\end{array}$ & 56.5 & 147 \\
\hline SOD1 & $\begin{array}{l}\text { CGCATGTTCCCAGACA } \\
\text { TCTA }\end{array}$ & $\begin{array}{l}\text { GAGCGGAAGATTGAGG } \\
\text { ATTG }\end{array}$ & 53.9 & 100 \\
\hline SOD2 & CTAGCCCGCTGACATT & $\begin{array}{l}\text { TTGCCCACATAGAAAT } \\
\text { GCAC }\end{array}$ & 54.5 & 101 \\
\hline GPX1 & $\begin{array}{l}\text { AGGCACAACAGTCAG } \\
\text { GGATT }\end{array}$ & $\begin{array}{l}\text { CAGGAACGCAAACAGA } \\
\text { GGG }\end{array}$ & 56.45 & 241 \\
\hline
\end{tabular}

208

A

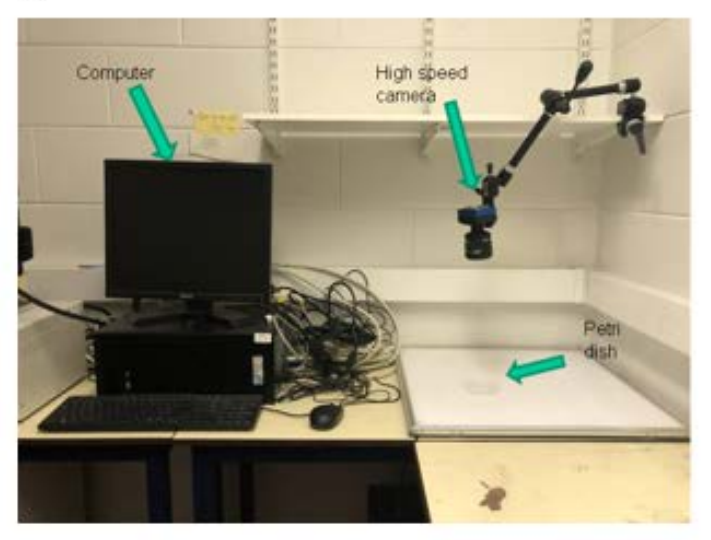

C

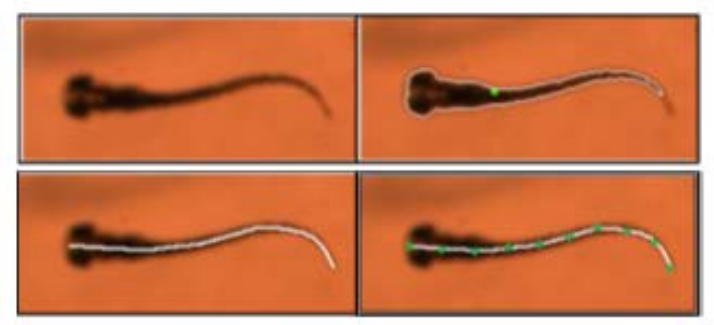

B

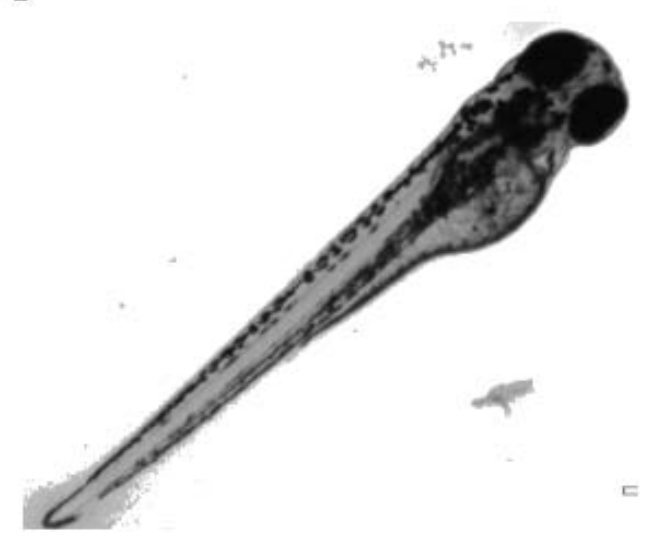

$\mathrm{D}$

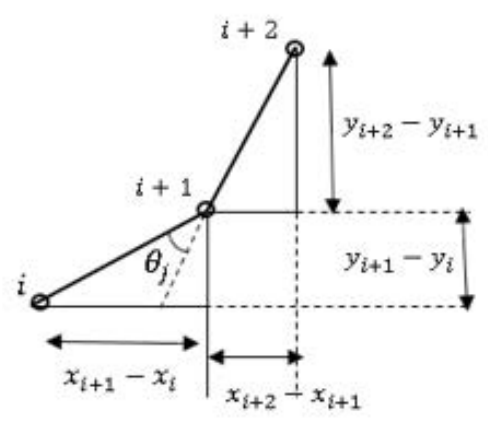


Fig. 1. Video recording and processing system and algorithm (A) Apparatus for video recording (B) Real zebrafish larvae picture taken from the lab (C) Key steps for estimating body deformation (D) Mathematical method for relative angle orientation extraction (segments are represented with straight lines).

\subsection{Computational fluid dynamics (CFD) solver \& kinematic motion solver}

A 3D 5-dpf zebrafish larva model was built with 51 ellipses extracted from the real fish silhouette and divided into nine segments (Fig 2A). In our previous research an independence test was carried out in order to determine the appropriate number of segments [29]. Density of the fish was assumed to be equal to water density, which is $1000 \mathrm{~kg} / \mathrm{m}^{3}$. The kinematic viscosity was set as $8.53 \mathrm{e}-7 \mathrm{~m}^{2} / \mathrm{s}$, which is water's kinematic viscosity at $27^{\circ} \mathrm{C}$. The flow field was numerically simulated using the open source CFD toolbox OpenFOAM version 3.0.x. The 3D computational domain shown in Fig 2B is 20 times the fish body length in the longitudinal (x) direction, 10 times the fish body length in the transverse (y) direction, and 4 times the fish body length in the perpendicular (z) direction. There is no incoming flow in the fluid domain. The fish started swimming from zero velocity. The boundary conditions are shown in Fig. 2B. Pressure boundary conditions are taken as zero gradient for all boundaries except the front and back plane, which were set as symmetry. Velocity boundary conditions for fish model were taken as 'movingWallVelocity' for all body segments and fixed value for the remaining patches to mimic the physical environment of the experiment. The movingWallVelocity is a special boundary condition applied in OpenFOAM, it was used for moving mesh cases and set the velocity to the desired value for moving walls. Unstructured mesh cells were distributed around fish model to tolerate the large internal mesh deformation during forward body motion, the head region was enlarged to be clearer and was shown in Fig 2C. Reynolds number was defined as $\frac{v l}{v}$, v stands for forward swimming velocity, $L$ is the body length of fish larva, and $v$ represented the kinematic viscosity mentioned above. For the entire simulation, Reynolds number was estimated to be 340 according to the desired forward velocity of zebrafish larvae, which stands for intermediate flow regime, this is consistent with the real fluid property of zebrafish larvae. 
The fluid domain was solved with incompressible Navier-Stokes equation written in Eqn 1, including the conservation equation of mass and momentum. In OpenFOAM, a customized solver PimpleDyMFoam was used to solve the transient, incompressible and single-phase Newtonian fluids. This solver was a combination of SIMPLE and PISO algorithm, which was suitable to deal with dynamic mesh motion.

$$
\nabla \bullet U^{\prime}=0
$$

$$
\frac{\partial}{\partial t} \rho \phi=-\nabla \bullet(\rho \stackrel{r}{U} \phi)+\nabla \bullet(D \nabla \phi)+S_{\phi}
$$

In the momentum conservation equation, time derivatives used $2^{\text {nd }}$ order implicit discretization scheme: CrankNicolson, and the pressure source term used cell-limited Gauss interpolation scheme to limit interpolated face values to improve boundedness and stability. Diffusion of transport used Gauss linear for interpolation of the diffusivity, and the convection term applied ReconCentral interpolation scheme. In OpenFOAM, velocity was stored at the cell centre, values were needed to be interpolated to the face centres linearly. ReconCentral interpolated the value in a different way that used extrapolated gradient-based correction from both sides onto the face, using $1 / 2$ weighting to increase stability for large deformation.

Solid body motion was processed with an open-source multibody dynamics analysis software MBDyn (https://www.mbdyn.org/). It solved initial value problem in the form of Differential-Algebraic Equations (DAE), integrated in time domain using A/L-stable multi-step integration schemes [48]. Constraints were added into the multi-body system to behave as a virtual joint to represent the muscles. In our case, the fitted sinusoidal-like functions were used as input in MBDyn to behave like constraints between two adjacent body segments to control their relative orientations. The differential-algebraic equations written in Eqn 2 were integrated with multi-step integration schemes in time domain implicitly. Finite element method was used to solve those algebraic equations. In this case, the whole fish body was represented with several constructed nodes in MBDyn (Fig 2D), these nodes included all the information about the body segments such as position and orientation. Relative 
269 orientations between each two nodes were constrained with specific types of joint to 270 constrain numbers of degrees of freedom. Relationship between nodes and joints was 271 shown in Fig.2E. MBDyn were coupled with OpenFOAM using the built-in 272 communication primitives, and kinematic data were transmitted bi-directionally. To 273 satisfy convergence criteria, a strong coupling, which enabled multi-step interactions 274 at each time value, was applied between the two software. Inter-process 275 communication was built with Transmission Control Protocol (TCP) socket. An 276 external force element in MBDyn allowed to communicate positions and orientations 277 of a set of nodes, and the corresponding linear and angular velocities with 278 OpenFOAM.

$$
\begin{gathered}
\mathrm{M}(\mathrm{x}) \&=\mathrm{q} \\
\dot{\mathrm{q}}+\phi_{/ \mathrm{x}}^{\mathrm{T}} \lambda=\mathrm{f}(\mathrm{x}, \dot{\mathrm{x}}, \mathrm{t}) \\
\phi(\mathrm{x}, \mathrm{t})=0
\end{gathered}
$$


A

B
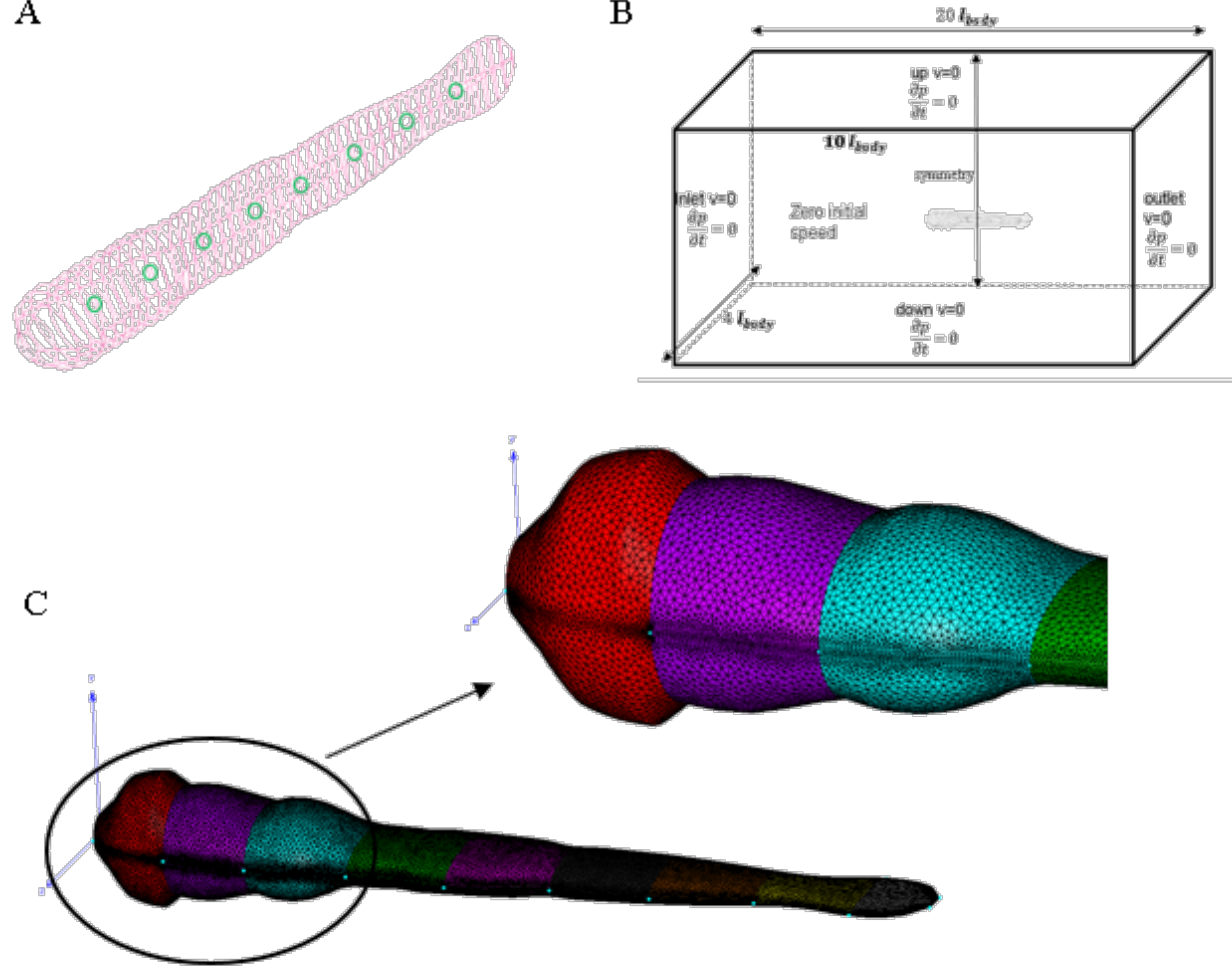

D<smiles>[3H]C([3H])[13CH3]</smiles>

0
$\mathrm{E}$

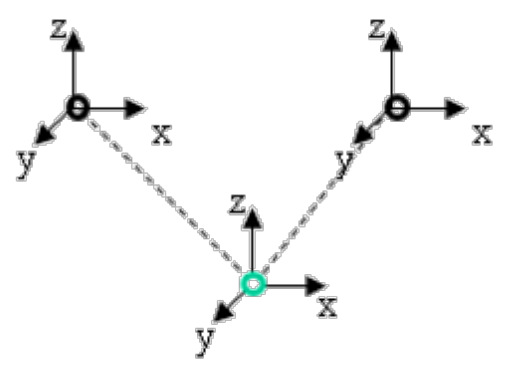

Fig. 2. (A) Constructed fish model with 51 ellipses; the entire body was divided into nine sections with eight joints. Joints are represented by green circles. (B) Initial fluid domain setup for a fish at rest. (C) Local mesh on fish model surface; head region is enlarged to be clearer. (D) Schematic diagram of 3-D fish model shown with constructed nodes in MBDyn with local frame. (E) Relationship between node and joint, black circles indicate node and joint is represented by green circle. The dashed line suggests that the position of node and joint can be coincident and the orientation can be different depending on the reference frame selected.

\subsection{Statistical analysis}

Statistical analysis was carried out using GraphPad Prism (V7.0 from GraphPad software Inc. San Diego, CA, USA) was used for statistical analysis. All multiple comparisons were performed using the one-way Anova with Bonferroni multiple comparison test. Statistical significance was considered when $p$ was $\leq 0.05$. 


\section{Results and discussion}

298

299

300

301

302

303

3.1 The effect of Gypenoside on swimming behaviour of zebrafish treated with acetic acid

Validation of our methodology has been made based on comparisons of experimentally-observed zebrafish forward-swimming velocity and tail beat angle with those results from numerical simulation in our previously published paper [29]. Head turning angle and tail beat angle in CFD simulations corresponded closely with experimental results, while a small discrepancy existed for velocity comparison. The latter could due to the fact that when the real fish swims, the changing body wave amplitude can lead to a velocity change, resulting in a higher experimentally calculated velocity compared to the numerical simulation. Swimming by fish larvae is powered by their axial muscle system [49]. For different fish species, the contribution of these muscles to swimming can vary. The power generated by muscle contraction bends the body and so interacts with the surrounding fluid. Muscle myotomes are aligned parallel to the longitudinal axis, with muscle fibers oriented about 40 degrees to the longitudinal axis of the fish [50]. Two types of muscles form the 3D structure on each side of the body. One type is white muscle, which powers the fish for fast starts such as escaping behaviors; the second type is red muscle that powers sustained swimming such as cyclic swimming [51]. During the larval stage, the white muscle is distributed predominantly in deeper regions below the skin and is enclosed in a thin outer layer of red muscle. Muscle contractions are driven by motoneurons in the spinal cord, and these contractions control the fish locomotion activity [52], therefore, muscle status can be reflected in activity level of the fish [9]. We compared the time spent by each group in three different swimming conditions: Inactive (when the fish was at rest or showed only a subtle tail beat with no obvious displacement in the water), Active 1 (when the fish swam cyclically for a relatively long period of time) and Active 2 (when the fish was swimming for a short period of time including acceleration with large tail curvature followed by a quick de-acceleration). As shown in Fig 3A, compared with control group, the GYP-treated group did not show any obvious changes with regard to the percentage of time spent in active swimming, 
indicating that $5 \mu \mathrm{g} / \mathrm{ml}$ GYP does not appear to be toxic to 5 dpf zebrafish larvae. Exposure to acetic acid resulted in the fish being inactive for $80 \%$ of the time. Inactivity decreased to approximately $50 \%$ of the time following GYP treatment of fish exposed to acetic acid, suggesting an alleviatory effect of GYP solution on the muscle inflammation caused by the acid. Cyclic swimming occurs randomly in $5 \mathrm{dpf}$ zebrafish larvae [47]; in the current study an increase of only $3 \%$ in time spent for cyclic swimming was observed after GYP treatment. However, for short time swimming, a significant increase of approximately $25 \%$ in total time is shown after GYP treatment. No obvious differences (less than 5\% in total time) are apparent between the GYP group and the GYP+AC group, suggesting that the GYP solution has an alleviatory effect on the muscle inflammation caused by $0.1 \%$ acetic acid.

Based on the simulated results from OpenFOAM such as position and orientation for each segment, the forward velocity of the fish larva was calculated from center of mass (COM) of the fish body. The COM was derived from the position of each segment at each time step using a mass-averaged method [44]. Fig 3B shows a mean velocity comparison for all groups. The mean velocity of cruising in the acetic acid group was significantly lower than that in each of the other three groups, while the GYP+AC group displayed a similar mean velocity to the GYP and the control groups. The statistical analysis in Fig 4A indicates that mean velocities of GYP and GYP+AC groups were significantly higher than that of the AC group, suggesting that the effect on forward velocity of exposure to $0.1 \%$ acetic acid was alleviated by $5 \mu \mathrm{g} / \mathrm{ml} \mathrm{GYP.}$

As the movement of each two body segments is constrained with a prescribed deformation equation, mechanical power distribution along the fish body can be approximated by power generated at the virtual joint between each two body segments. The virtual joint behaves like fish muscle distributed along the body to generate mechanical power. The mechanical power generated from fish locomotion includes the translational power due to linear motion and the rotational power due to body rotation [29]. As the fish is moving cyclically, all the other terms are cancelled out except for the rotational power. Therefore, the mechanical power was estimated 
with the cross product of torque and angular velocity, as shown in equation 3(a), while the total power transmitted into the water was calculated with equation 3(b). During cruising, the change of total kinetic energy equals zero, which means the absolute value of work done externally and internally are the same, thus the hydrodynamic power equals mechanical power in absolute value (shown as equation 3(c)).

$$
\begin{gathered}
P_{M}=\sum_{j} M_{i} g \omega_{i} \\
P_{H}=\sum_{j}-F_{j} g V_{j} \\
\overline{P_{M}}=\overline{P_{H}}
\end{gathered}
$$

In the above equation, $P_{M}$ is the mechanical power of fish muscle, while $P_{H}$ represents hydrodynamic power generated by interactions with surrounding fluid. $M_{i}$ is the internal torque for the $i^{\text {th }}$ joint calculated by MBDyn in the global frame, $\omega_{i}$ represents the angular velocity for the $i^{\text {th }}$ joint. $F_{j}$ is the hydrodynamic force acting on the $j^{\text {th }}$ body, while $V_{j}$ represents the $j^{\text {th }}$ body velocity. Fig. $3 \mathrm{C}$ compares the total hydrodynamic power exerted on the entire body of the control group with the drug-treated groups. It was not surprising that the tendency is similar to forward velocity as the hydrodynamic power was calculated based on the forward velocity of the fish. As we expected, $5 \mu \mathrm{g} / \mathrm{ml} \mathrm{GYP}$ could alleviate the influences on power generation, allowing the muscle of the zebrafish larvae to generate greater power, producing larger body deformation and larger hydrodynamic power compared with the $0.1 \%$ acetic acid treated group. The statistical analysis of total hydrodynamic power shown in Fig 4B makes the conclusion more persuasive. There was a significant difference in hydrodynamic power before and after GYP treatment with acetic acid; as the muscle correlated with the power supply, it was prudent to deduce that GYP treatment could to some extent protect fish muscle from inflammation caused by acetic acid. Unlike many biomechanical situations in which the propulsive system is separate from the main body, zebrafish larvae undulate their entire body to swim forward [53], therefore the whole body contributes to the generation of thrust and drag during the tail beat cycle, although the contribution from each might differ. 
In Fig 3D and Fig 3E, we compared the distribution of hydrodynamic power and mechanical power along the fish body to quantify the differences of force and power for different groups. To display the power distribution more clearly, we have used absolute values for hydrodynamic power distribution along the body. Given that the fish larvae were submerged in the solution, the whole body would have been exposed, therefore we assumed that the axial muscle along the entire body would be affected by exposure to acetic acid and GYP. Although the total power is kept balanced during cruising, the power distribution is different for the internal muscle and body surface. The averaged hydrodynamic power for the fish larvae in different groups in Fig 3D showed a significant higher value starting from approximately $75 \%$ of body length. According to motion equations, this region had the largest shape change along the body in global frame, resulting in larger fluid force and more hydrodynamic power. In Fig.3E, the mechanical power generated along the body showed an increase towards the tail and a steep decrease at the tail. Higher mechanical power starts from approximately joint number 4 , located at the centre of the body, indicating that the main power is generated in the posterior half of the body. In the posterior region, the larger body curvature indicates higher muscle strain, thus indicating that more strenuous work is done by this part of the body. Among the different groups, the group treated with $0.1 \%$ acetic acid displayed significantly lower hydrodynamic power and mechanical power. After treatment with GYP, the power increased to a level close to that of the control group. For all body segments, both mechanical and hydrodynamic power followed the tendency of the total hydrodynamic power, suggesting that exposure influenced the entire axial muscle system. In this part of the study, the internal muscle power has been quantified to provide a better understanding of the beneficial effects of GYP. 

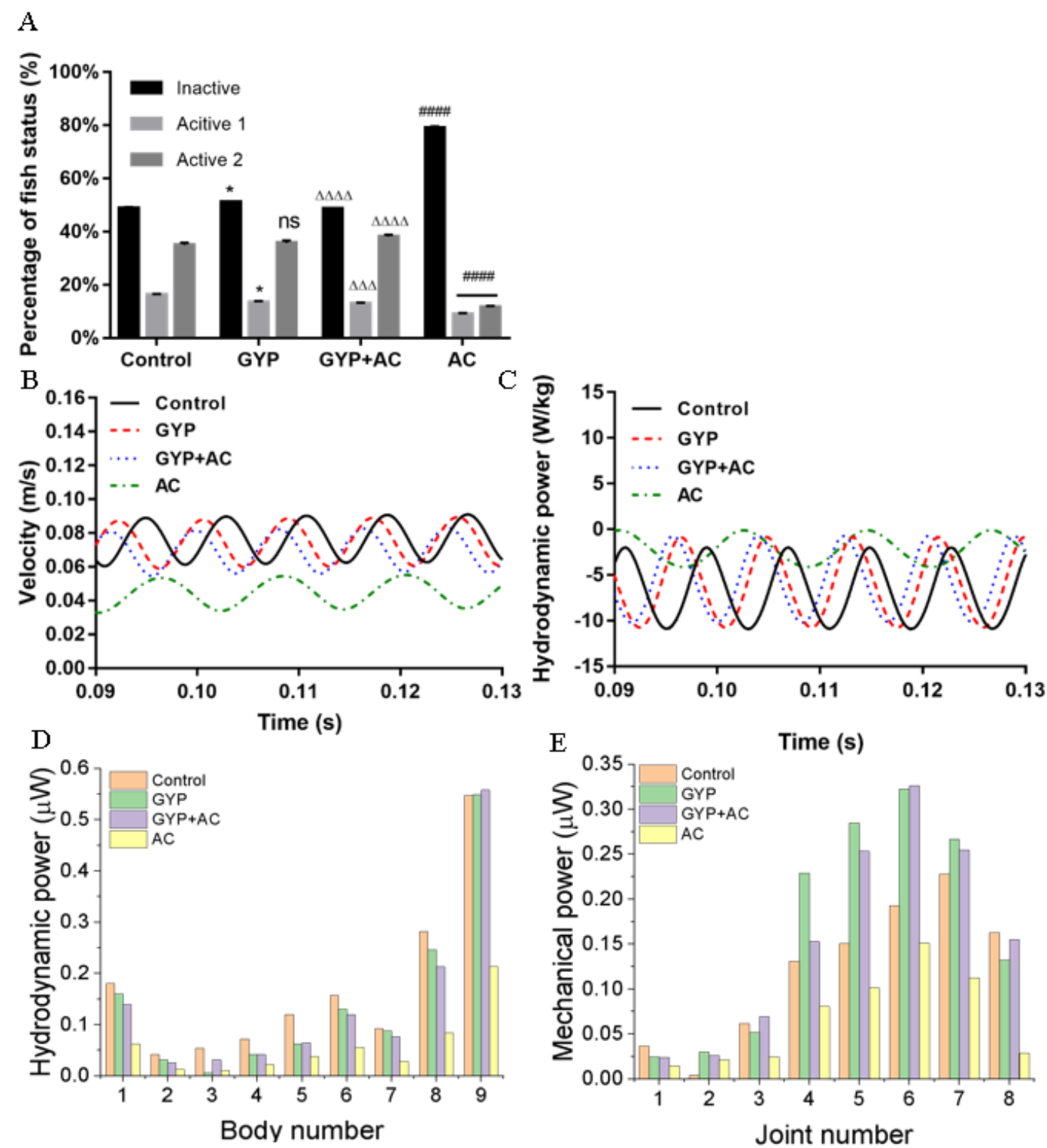

Fig. 3. (A) Comparisons of swimming status for each treatment group, with mean \pm . s.d. ${ }^{*} p<0.05,{ }^{* *} p<0.01,{ }^{* * *} p<0.001,{ }^{* * * *} \mathrm{p}<0.0001$, ns for not significant. Comparison of GYP group with control group is represented by '*'; comparison of AC treated group with GYP+AC group is represented by ' $\Delta$ '; comparison of control with AC group is represented by '\#’. AC: 0.1\% acetic acid; GYP: Gypenosides. Inactive (black column): Active 1: cyclic swimming (light grey) Active 2 (dark grey): short time swimming. (B) Velocity comparison for four groups based on numerical simulations. (C) Total hydrodynamic power comparison for four groups. The negative values indicate balance with positive mechanical power due to the zero total kinetic energy. (D) Hydrodynamic power distribution along fish body. Power exerted by the fluid is calculated for each body segments, expressed with body numbering from 1-9. (E) Mechanical power distribution along fish body. Power generated by all virtual joints numbering from 1-8 between two adjacent body segments are calculated. 


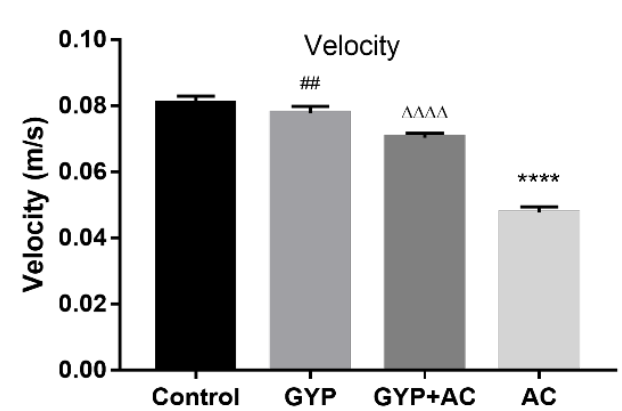

A

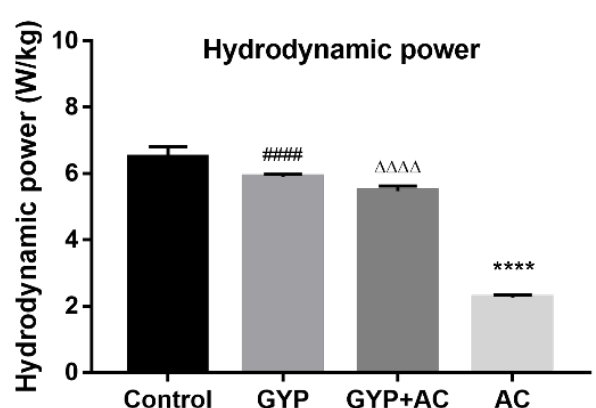

B
425

426

427

428

429

430

431

432

433

434

435

436

437

438

439

440

441

442

443

444

We also carried out statistical analysis of hydrodynamic and mechanical power at different body sections and virtual joints to clarify if the effects of GYP protection varied along the zebrafish body (Fig. 5). For hydrodynamic power, the overall tendency along the body of power generated remained the same with total body hydrodynamic power shown in Fig. 4B. It was obvious that the zebrafish larvae exposed to acetic acid generated lower hydrodynamic power. When comparing GYP and GYP+AC group, it is possible that at some body sections the GYP+AC group zebrafish can generate higher hydrodynamic power than that of GYP group. This might be caused by minor side effects of GYP, together with stimulation by acetic acid, as the effect of GYP could vary at different body sections due to different absorbing abilities. However, similar circumstances should not affect mechanical power, which directly reflects power generated with muscle contractions. 

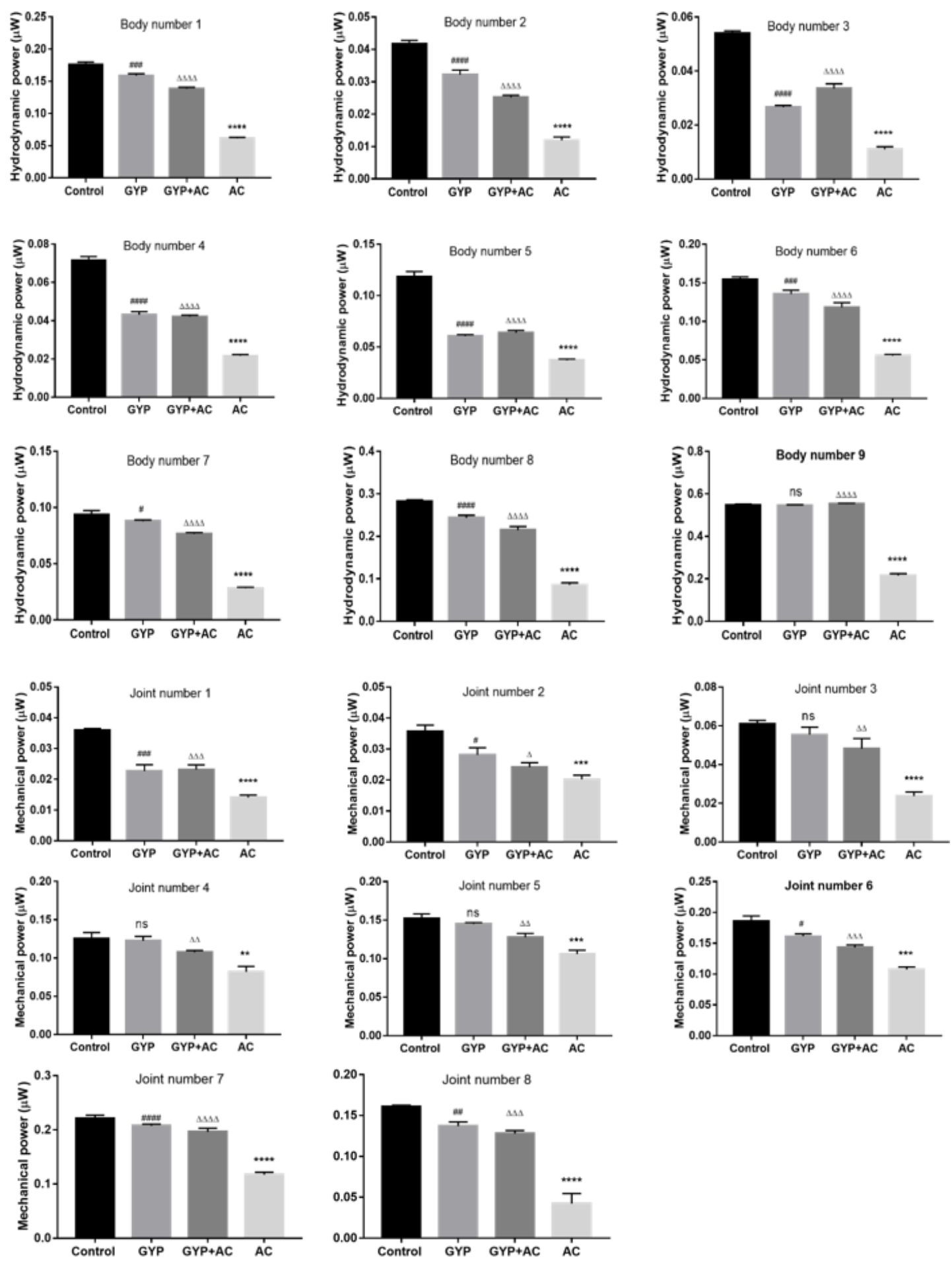

Fig. 5. Statistical analysis on hydrodynamic and mechanical power along the body. Body sections numbering from 1 to 9 depict hydrodynamic power comparisons among treated groups. Virtual joints numbering from 1 to 8 display mechanical power comparisons among treated groups. For all groups, ns, no significance; ${ }^{*} p<0.05$, ${ }^{* *} p<0.01$, ${ }^{* * *} p<0.001,{ }^{* * * *} p$ $<0.0001$. Comparison of Control group with GYP group is represented by '\#'; comparison of Control with AC is represented by '*'; and comparison of GYP+AC with AC is represented by ' $\Delta$ '. 
A possible factor that can influence fish swimming performance is body elasticity, which has not been considered in the current research. Zebrafish larvae swim in an intermediate flow regime with large viscous effects, which requires higher thrust to overcome drag; this is achieved by higher tail beat frequency and amplitude. This would in turn cause higher drag and energy loss (lateral momentum shedding), and so any evaluation of swimming performance should consider the body elasticity, which can save energy. [54] studied flexural stiffness of superficial neuromasts as this is correlated with the detection of surrounding fluid. [55] provided a prediction of fish body's visco-elastic properties and related it to muscle mechanical behaviour in vivo based on a continuous beam model. Although not tested on zebrafish specifically, fish in general tend to adjust their body elasticity at specific positions in order to optimize their swimming performance by increasing efficiency and energy saving [56]. However, the distribution of visco-elastic properties along the fish body are difficult to measure precisely, thus the mutual contributions from visco-elastic properties to the optimized swimming performance cannot be determined individually. Moreover, it is technically difficult to observe subtle body curvature changes. Different fish species may have different elasticity for different purposes, such as for acceleration/deceleration or cruising swimming [21]. Acknowledging the importance of body elasticity for a better understanding of muscle function in controlling fish swimming, we intend to focus in our future research on the visco-elastic properties at predicted positions, with the help of muscle dissection. To be specific, muscle related adverse medical treatment may have effects on muscle tissues such as shortened or dissolved local muscle fibres [57]. By applying predicted elasticity characteristics and comparing these with the live fish tissue properties at those locations, it might be possible to account for the influences on altered swimming behaviours.

\subsection{Gypenoside increased anti-oxidative capacity and decreased inflammation in} acetic acid-treated zebrafish larvae

Oxidative stress and inflammation play an important role in the development of pain and have been shown to be two main molecular mechanisms involved in muscle atrophy in mice and also in humans $[58,59]$. This suggests they might be 
capable of inducing muscle-related symptoms. Previous work showed that the natural product quercetin inhibited inflammatory pain by increasing glutathione (GSH) generation and decreasing the production of inflammatory mediators [60]. Another natural product, diosgenin, demonstrated a capacity to ameliorate the neuropathic pain associated with diabetes mellitus. Diosgenin treatment in streptozotocin-induced diabetic rat inhibited production of IL-1 $\beta$ and TNF- $\alpha$ in serum, enhanced catalase and SOD activities in serum, sciatic nerve and dorsal root ganglion, and restored nociceptive thresholds [61]. Our previous work demonstrated that GYP restored antioxidative capacity and inhibited proinflammatory cytokine production in $\mathrm{H}_{2} \mathrm{O}_{2}$-treated retinal pigment epithelial (RPE) cells [33]. Intraperitoneal injection of diluted acetic acid has been widely used to induce pain in rodent models [60,62]. Acetic acid induces production of inflammatory cytokines such as IL-1 $\beta$, IL-8 and TNF- $\alpha$, which mediate writhing response in mice [62]. Acetic acid has been applied to induce pain and nociception in zebrafish (larvae or adults) via introduction to zebrafish water or by local injection [10, 19, 41, 63-65]. Studies on functional consequence of acetic acid exposure in zebrafish have mainly been focused on zebrafish locomotion activity and behavior. Here we examined the effects of acetic acid on oxidative stress and inflammation in zebrafish and whether GYP mediated oxidative stress and inflammation in acetic acid-treated zebrafish larvae (shown as Fig. 6). QRT-PCR data demonstrated that expression of antioxidant genes, including SOD1, SOD2 and GPX1, was significantly decreased in acetic acid-treated zebrafish compared to untreated control zebrafish and that co-treatment with GYP resulted in a marked increase in expression of these three genes (shown as Fig. 6A). Acetic acid treatment caused significantly increased expression of inflammatory cytokine genes IL-1 $\beta$, IL-6 and TNF- $\alpha$ when compared to untreated control zebrafish; co-treatment with GYP reversed the acetic acid-induced effects (shown as Fig. 6B). Based on our numerically simulated results regarding internal muscle mechanism variations, we deduce that GYP can alleviate pain caused by $0.1 \%$ acetic acid and the effect can be attributed to antioxidant and anti-inflammatory functions and expressed with 
quantified internal muscle mechanisms. Most importantly, we have developed a novel approach to evaluate the therapeutic potential of GYP for neuropathic pain.
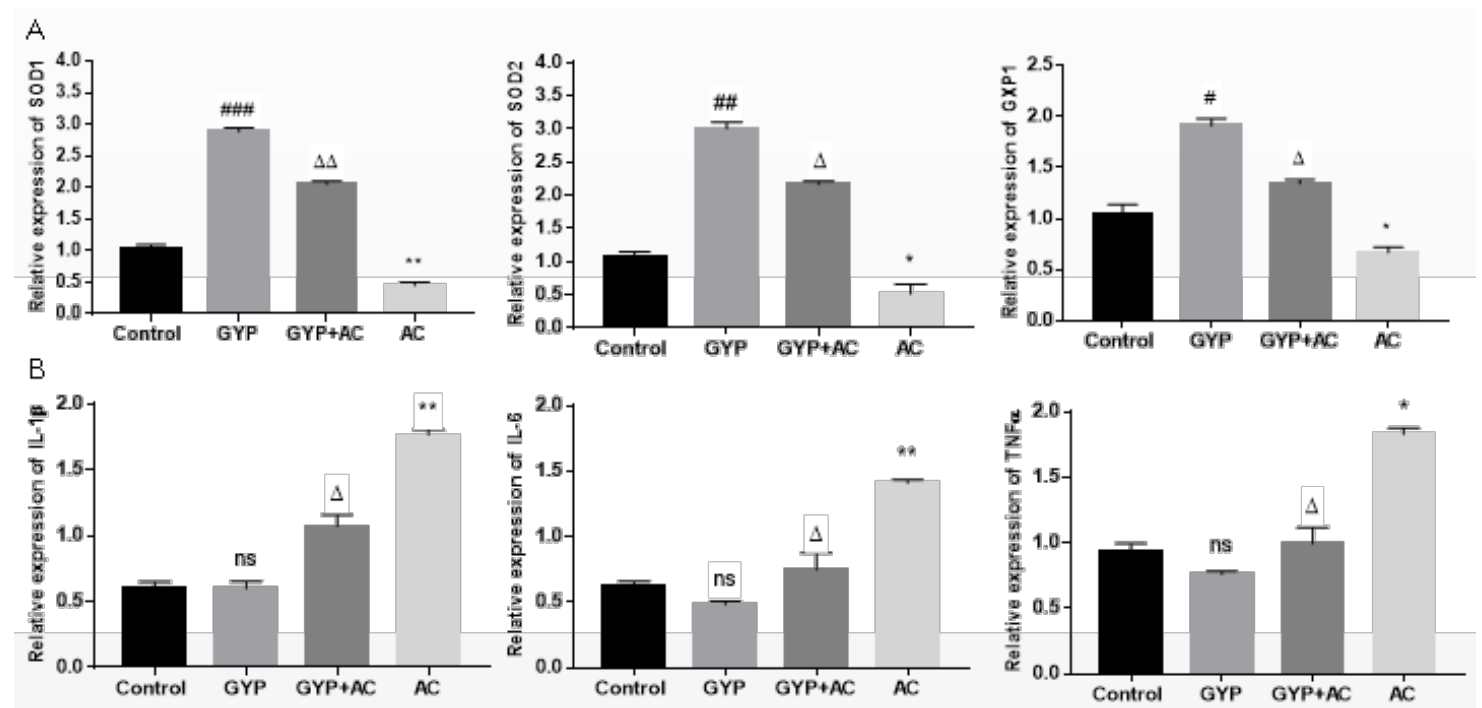

Fig. 6. GYP regulated expression of antioxidant and pro-inflammatory genes. (A) Expression of antioxidant genes in untreated and treated zebrafish larvae. (B) Expression of proinflammatory genes. Experiments were repeated three times. Data are presented as means \pm standard error (SE). ns, no significance; ${ }^{*} p<0.05,{ }^{* *} p<0.01,{ }^{* * *} p<0.001$. Comparison of Control group with GYP group is represented by '\#'; comparison of Control with AC is represented by '*'; and comparison of GYP+AC with AC is represented by ' $\Delta$ '.

In conclusion, in this paper an analysis of the protective effect of Gypenosides against acetic acid on zebrafish larvae has been carried out. Unlike previous research, we have combined CFD simulations on zebrafish locomotion to study the effects on zebrafish behaviors and internal muscle mechanics. As a high concentration of acetic acid is known to cause pain/damage to zebrafish larvae muscle and has been tested extensively, we chose to investigate the protective effects of of $5 \mu \mathrm{g} / \mathrm{mL}$ GYP against exposure to $0.1 \%$ acetic acid and observed the alleviation of muscle inflammation after GYP treatment. The conclusions have been confirmed by both QRT-PCR data and CFD simulated results, showing that our computational method could assist in evaluating the protective effect of GYP against acetic acid and other harmful substances. In addition, we have also quantified the internal muscle mechanics that can partially reflect the effects of medicine on muscle status, data that is difficult to acquire from standard experiments. Considering the cheaper cost and 
faster preparations of CFD simulation compared to qRT-PCR analysis, our method could potentially be used to evaluate the effects of drugs on zebrafish behaviors and thus support the development of therapeutic drugs for neuropathic pain.

Author contribution

Q.X. and X.S. conceived the study. Z.Z. and G.M.T. carried out the experiments. Z.Z., G.M.T., H.J. and X.S. analysed the data. Z.Z., Q.X., J.R. and X.S. wrote the manuscript.

\section{Acknowledgements}

This work was partially supported by the Rosetrees Trust (M160, M160-F1, M160-F2) and the Lotus Scholarship Program of Hunan Province (2019), China. X.S. is a Visiting Professor to Shaoyang University.

Conflicts of interest

The authors have no interest to declare.

\section{References}

1. Lieschke, G.J. and P.D. Currie, Animal models of human disease: zebrafish swi m into view. Nature Reviews Genetics, 2007, 8,353-367,https://doi.org/10.1038/n rg2091

2. Markovich, M.L., N.V. Rizzuto, and P.B. Brown, Diet Affects Spawning in Zeb rafish. Zebrafish, 2007, 4,69-74,https://doi.org/10.1089/zeb.2006.9993

3. Schaeck, M., K. Van den Broeck W Fau - Hermans, A. Hermans K Fau - De costere, and A. Decostere, Fish as research tools: alternatives to in vivo exper iments. Alternatives to Laboratory Animals, 2013, 41,https://doi.org/10.1177/0261 19291304100305

4. Malafoglia, V., B. Bryant, W. Raffaeli, A. Giordano, and G. Bellipanni, The ze brafish as a model for nociception studies. Journal of Cellular Physiology, 201 3, 228,1956-1966, https://doi.org/10.1002/jcp.24379

5. Gregory, N.S., C.R. Harris Al Fau - Robinson, P.M. Robinson Cr Fau - Doug herty, P.N. Dougherty Pm Fau - Fuchs, K.A. Fuchs Pn Fau - Sluka, and K.A. Sluka, An overview of animal models of pain: disease models and outcome $\mathrm{m}$ easures. Journal of Pain, 2013, 14,1255-1269,https://doi.org/10.1016/j.jpain.2013. $\underline{06.008}$

6. M.D., J.C.M., J.W.B. P.A.-C., M.K. Borden, K.M. Lorenz, and N.A. Ross, Nat ural antiinflammatory agents for pain relief in athletes. Journal of Neurosurger y, 2006, 21,https://doi.org/10.3171/foc.2006.21.4.12 
602

603

604

605

606

607

608

609

610

611

612

613

614

615

616

617

618

619

620

621

622

623

624

625

7. Curtright, A., M. Rosser, S. Goh, B. Keown, E. Wagner, J. Sharifi, D.W. Raib le, and A. Dhaka, Modeling Nociception in Zebrafish: A Way Forward for Un biased Analgesic Discovery. PLOS ONE, 2015, 10,e0116766,https://doi.org/10.13 71/journal.pone.0116766

8. Wolman, M. and M. Granato, Behavioral genetics in larval zebrafish: learning from the young. Developmental Neurobiology, 2011, 72,366-372,https://doi.org/ 10.1002/dneu.20872

9. Correia, A.D., S.R. Cunha, M. Scholze, and E.D. Stevens, A Novel Behavioral Fish Model of Nociception for Testing Analgesics. Pharmaceuticals, 2011, 4,66 5-680,https://doi.org/10.3390/ph4040665

10. Taylor, J.C., L.S. Dewberry, S.K. Totsch, L.R. Yessick, J.J. DeBerry, S.A. Watt s, and R.E. Sorge, A novel zebrafish-based model of nociception. Physiology \& Behavior, 2017, 174,83-88,https://doi.org/10.1016/j.physbeh.2017.03.009

11. Li, G., U.K. Müller, J.L. van Leeuwen, and H. Liu, Body dynamics and hydro dynamics of swimming fish larvae: a computational study. Journal of Experime ntal Biology, 2012, 215,4015,https://doi.org/10.1242/jeb.071837

12. Sanchez-Simon, F.M., F.J. Arenzana, and R.E. Rodriguez, In vivo effects of mo rphine on neuronal fate and opioid receptor expression in zebrafish embryos.

European Journal of Neuroscience, 2010, 32,550-559,https://doi.org/10.1111/j.146 0-9568.2010.07317.x

13. Santos, L.C., J. Ruiz-Oliveira, P.F. Silva, and A.C. Luchiari, Caffeine Dose-Res ponse Relationship and Behavioral Screening in Zebrafish, in The Question of Caffeine. 2017.

14. Sneddon, L.U., The evidence for pain in fish: the use of morphine as an anal gesic. Applied Animal Behaviour Science, 2003, 83,153-162,https://doi.org/10.10 16/S0168-1591(03)00113-8

15. Abdelkader, T.S., T.-H. Chang Sn Fau - Kim, J. Kim Th Fau - Song, D.S. So ng J Fau - Kim, J.-H. Kim Ds Fau - Park, and J.H. Park, Exposure time to c affeine affects heartbeat and cell damage-related gene expression of zebrafish Danio rerio embryos at early developmental stages. Journal of Applied Toxicol ogy, 2012, 33,1277-1283,https://doi.org/10.1002/jat.2787

16. Rana, N., A. Moond M Fau - Marthi, S. Marthi A Fau - Bapatla, T. Bapatla S Fau - Sarvepalli, K. Sarvepalli T Fau - Chatti, A.K. Chatti K Fau - Challa, and A.K. Challa, Caffeine-induced effects on heart rate in zebrafish embryos and possible mechanisms of action: an effective system for experiments in che mical biology. Zebrafish, 2010, 7,https://doi.org/10.1089/zeb.2009.0631

17. Lopez-Luna, J., Q. Al-Jubouri, W. Al-Nuaimy, and L.U. Sneddon, Reduction in activity by noxious chemical stimulation is ameliorated by immersion in analg esic drugs in zebrafish. Journal of Experimental Biology, 2017, 220,1451, https:/ /doi.org/10.1242/jeb.146969

18. Lopez-Luna, J.A.-O., Q. Al-Jubouri, W. Al-Nuaimy, and L.U. Sneddon, Impact of stress, fear and anxiety on the nociceptive responses of larval zebrafish. $\mathrm{P}$ LOS ONE, 2017e0181010,https://doi.org/10.1371/journal.pone.0181010

19. Steenbergen, P.J. and N. Bardine, Antinociceptive effects of buprenorphine in ze 
brafish larvae: An alternative for rodent models to study pain and nociception? Applied Animal Behaviour Science, 2014, 152,92-99,https://doi.org/10.1016/j.ap planim.2013.12.001

20. Voesenek, C.J., R.P.M. Pieters, and J.L. van Leeuwen, Automated Reconstructio $n$ of Three-Dimensional Fish Motion, Forces, and Torques. PLOS ONE, 2016, 11,e0146682,https://doi.org/10.1371/journal.pone.0146682

21. Eric.D.Tytell, C.-Y. Hsu, and T.L. Williams, Interactions between internal force $s$, body stiffness, and fluid environment in a neuromechanical model of Lampre y swimming. Biophyics and Computational Biology, 201010.1073/pnas.10115641 07/-/DCSupplemental

22. McMillen, T. and P. Holmes, An elastic rod model for anguilliform swimming. Journal of Mathematical Biology, 2006, 53,843-886,https://doi.org/10.1007/s002 85-006-0036-8

23. Voesenek, C.J., F.T. Muijres, and J.L. van Leeuwen, Biomechanics of swimmin $g$ in developing larval fish. Journal of Experimental Biology, 2018, 221,jeb149 583,https://doi.org/10.1242/jeb.149583

24. J. Lighthill, M., Large-Amplitude Elongated-Body Theory of Fish Locomotion. Vol. 179. 1971. 125-138.

25. Carling, J., T.L. Williams, and G. Bowtell, Self-propelled anguilliform swimmin $g$ : simultaneous solution of the two-dimensional navier-stokes equations and $\mathrm{Ne}$ wtons laws of motion. Journal of Experimental Biology, 1998, 201,3143,980883 0

26. Kern, S. and P. Koumoutsakos, Simulations of optimized anguilliform swimming. Journal of Experimental Biology, 2006, 209,4841,https://doi.org/10.1242/jeb.025 26

27. Borazjani, I. and F. Sotiropoulos, Numerical investigation of the hydrodynamics of carangiform swimming in the transitional and inertial flow regimes. Journa l of Experimental Biology, 2008, 211,1541,https://doi.org/10.1242/jeb.015644

28. Borazjani, I. and F. Sotiropoulos, Numerical investigation of the hydrodynamics of anguilliform swimming in the transitional and inertial flow regimes. Journa l of Experimental Biology, 2009, 212,576,https://doi.org/10.1242/jeb.025007

29. Zhao, Z., G. Li, Q. Xiao, H.-R. Jiang, G.M. Tchivelekete, X. Shu, and H. Liu, Quantification of the influence of drugs on zebrafish larvae swimming kinema tics and energetics. PeerJ, 2020, 8,e8374,https://doi.org/10.7717/peerj.8374

30. Hsu, H.Y., K.-W. Yang Js Fau - Lu, C.-S. Lu Kw Fau - Yu, S.-T. Yu Cs Fau - Chou, J.-J. Chou St Fau - Lin, Y.-Y. Lin Jj Fau - Chen, M.-L. Chen Yy F au - Lin, F.-S. Lin Ml Fau - Chueh, S.-S. Chueh Fs Fau - Chen, J.-G. Chen Ss Fau - Chung, and J.G. Chung, An experimental study on the antileukemia e ffects of gypenosides in vitro and in vivo. Integrative Cancer Therapies, 2011, 101,12,https://doi.org/10.1177/1534735410377198

31. Dong, S.-Q., Q.-P. Zhang, J.-X. Zhu, M. Chen, C.-F. Li, Q. Liu, D. Geng, an d L.-T. Yi, Gypenosides reverses depressive behavior via inhibiting hippocampa I neuroinflammation. Biomedicine \& Pharmacotherapy, 2018, 106,1153-1160, http s://doi.org/10.1016/j.biopha.2018.07.040 
32. Zhang, H.-K., Y. Ye, Z.-N. Zhao, K.-J. Li, Y. Du, Q.-M. Hu, and J.-F. He, Ne uroprotective effects of gypenosides in experimental autoimmune optic neuritis. International journal of ophthalmology, 2017, 10,541-549, https://doi.org/10.1824 $\underline{0 / \mathrm{ijo} 0.2017 .04 .07}$

33. Alhasani, R.H., L. Biswas, A.M. Tohari, X. Zhou, J. Reilly, J.F. He, and X. S $\mathrm{hu}$, Gypenosides protect retinal pigment epithelium cells from oxidative stress. Food and Chemical Toxicology, 2018, 112,72-85,https://doi.org/10.1016/j.fct.2017. 12.037

34. Li, L., B.H. Jiao L Fau - Lau, and B.H. Lau, Protective effect of gypenosides against oxidative stress in phagocytes, vascular endothelial cells and liver mic rosomes. Cancer Biotherapy and Radiopharmaceuticals, 1993, 263,72, https://doi.o rg/10.1089/cbr.1993.8.263

35. Megalli, S., N.M. Aktan F Fau - Davies, B.D. Davies Nm Fau - Roufogalis, a nd B.D. Roufogalis, Phytopreventative anti-hyperlipidemic effects of gynostemm a pentaphyllum in rats. Journal of Pharmacy and Pharmaceutical Sciences, 200 5, 507,15,16401396

36. Quan, Y. and M.Z. Qian, Effect and mechanism of gypenoside on the inflamm atory molecular expression in high-fat induced atherosclerosis rats. Zhongguo Zhong Xi Yi Jie He Za Zhi, 2010, 403,6,20669679

37. Mettam, J.J., L.J. Oulton, C.R. McCrohan, and L.U. Sneddon, The efficacy of $t$ hree types of analgesic drugs in reducing pain in the rainbow trout, Oncorhyn chus mykiss. Applied Animal Behaviour Science, 2011, 133,265-274, https://doi.0 rg/10.1016/j.applanim.2011.06.009

38. Reilly, S.C., A.R. Quinn Jp Fau - Cossins, L.U. Cossins Ar Fau - Sneddon, a nd L.U. Sneddon, Novel candidate genes identified in the brain during nocicep tion in common carp (Cyprinus carpio) and rainbow trout (Oncorhynchus myki ss). Neuroscience Letters, 2008, 135,https://doi.org/10.1016/j.neulet.2008.03.075

39. Bingham, S., P.J. Beswick, D.E. Blum, N.M. Gray, and I.P. Chessell, The role of the cylooxygenase pathway in nociception and pain. Seminars in Cell \& D evelopmental Biology, 2006, 17,544-554,https://doi.org/10.1016/j.semcdb.2006.09. 001

40. Diggles, B.K., R. Arlinghaus, H.I. Browman, S.J. Cooke, I.G. Cowx, A.O. Kas umyan, B. Key, J.D. Rose, W. Sawynok, A. Schwab, A.B. Skiftesvik, E.D. Ste vens, C.A. Watson, and C.D.L. Wynne, Responses of larval zebrafish to low $p$ $\mathrm{H}$ immersion assay. Comment on Lopez-Luna et al. Journal of Experimental Bi ology, 2017, 220,3191,https://doi.org/10.1242/jeb.162834

41. Lopez-Luna, J., Q. Al-Jubouri, W. Al-Nuaimy, and L.U. Sneddon, Reduction in activity by noxious chemical stimulation is ameliorated by immersion in analg esic drugs in zebrafish. The Journal of Experimental Biology, 2017, 220,14511458,10.1242/jeb.146969

42. Yu, H., L. Shi, G. Qi, S. Zhao, Y. Gao, and Y. Li, Gypenoside Protects Cardi omyocytes against Ischemia-Reperfusion Injury via the Inhibition of Mitogen-Ac tivated Protein Kinase Mediated Nuclear Factor Kappa B Pathway In Vitro an d In Vivo. Frontiers in pharmacology, 2016, 7,148-148,https://doi.org/10.3389/fph 
ar.2016.00148

43. Huang, T.H., V. Li Y Fau - Razmovski-Naumovski, V.H. Razmovski-Naumovsk i V Fau - Tran, G.Q. Tran Vh Fau - Li, C.C. Li Gq Fau - Duke, B.D. Duke Cc Fau - Roufogalis, and B.D. Roufogalis, Gypenoside XLIX isolated from G ynostemma pentaphyllum inhibits nuclear factor-kappaB activation via a PPARalpha-dependent pathway. Journal of Biomedical Science, 2006https://doi.org/10. 1007/s11373-006-9076-8

44. van Leeuwen, J.L., C.J. Voesenek, and U.K. Müller, How body torque and Str ouhal number change with swimming speed and developmental stage in larval zebrafish. Journal of The Royal Society Interface, 2015, 12,20150479,https://doi. org/10.1098/rsif.2015.0479

45. Webb, P.W., Form and Function in Fish Swimming. Scientific American, 1984, 251,72-83,https://www.jstor.org/stable/24969414

46. Sancho, G., D. Ma, and P. Lobel, Behavioral observations of an upcurrent reef colonization event by larval surgeonfish Ctenochaetus strigosus (Acanthuridae). Marine Ecology Progress Series, 1997, 153,311-315,10.3354/meps153311

47. Muller, U.K., Swimming of larval zebrafish: ontogeny of body waves and impli cations for locomotory development. The Journal of Experimental Biology, 200 4, 207,853-868,10.1242/jeb.00821

48. Li, Y., Coupled Computational fluid dynamics/multibody dynamics method with application to wind turbine simulations. Lowa Research online, 2014

49. Voesenek, C.J., R.P. Pieters, and J.L. van Leeuwen, Automated Reconstruction of Three-Dimensional Fish Motion, Forces, and Torques. PLoS One, 2016, 11,e 0146682,10.1371/journal.pone.0146682 PMC4713831

50. Altringham, J.D. and D.J. Ellerby, Fish swimming: patterns in muscle function. Journal of Experimental Biology, 1999, 202,3379-3403,10562522

51. Dou, Y., M. Andersson-Lendahl, and A. Arner, Structure and Function of Skele tal Muscle in Zebrafish Early Larvae. Journal of General Physiology, 2008, 13 1,445-453,https://doi.org/10.1085/jgp.200809982

52. Ekeberg, Ö., S. Grillner, and A. Lansner, The Neural Control of Fish Swimmin g Studied Through Numerical Simulations. Adaptive Behavior, 1995, 3,363-384, https://doi.org/10.1177/105971239500300402

53. Zhao, W., A. Ming, and M. Shimojo, Development of High-Performance Soft Robotic Fish by Numerical Coupling Analysis. Applied Bionics and Biomechani cS, 2018, 2018,12,https://doi.org/10.1155/2018/5697408

54. McHenry, M.J. and S.M. van Netten, The flexural stiffness of superficial neuro masts in the zebrafish (\&lt;em\&gt;Danio rerio\&lt;/em\&gt;) lateral line. Journal of Experimental Biology, 2007, 210,4244,https://doi.org/10.1242/jeb.009290

55. Zhang, W., Y. Yu, and B. Tong, Prediction of fish body's passive visco-elastic properties and related muscle mechanical performance in vivo during steady $s$ wimming. Science China Physics, Mechanics and Astronomy, 2014, 57,354-364, https://doi.org/10.1007/s11433-013-5372-2

56. Tytell, E.D., M.C. Leftwich, C.-Y. Hsu, B.E. Griffith, A.H. Cohen, A.J. Smits, C. Hamlet, and L.J. Fauci, Role of body stiffness in undulatory swimming: In 
sights from robotic and computational models. Physical Review Fluids, 2016, 1, 073202,https://doi.org/10.1103/PhysRevFluids.1.073202

57. Lin, Y.-Y., Muscle diseases in the zebrafish. Neuromuscular Disorders, 2012, 2 2,673-684,https://doi.org/10.1016/j.nmd.2012.04.007

58. Salim, S., Oxidative Stress and the Central Nervous System. The Journal of ph armacology and experimental therapeutics, 2017, 360,201-205,https://doi.org/10.1 124/jpet.116.237503

59. Keeble, J.E., L. Bodkin Jv Fau - Liang, R. Liang L Fau - Wodarski, M. Wod arski R Fau - Davies, E.S. Davies M Fau - Fernandes, C.d.F. Fernandes Es F au - Coelho, F. Coelho Cde F Fau - Russell, R. Russell F Fau - Graepel, M. N. Graepel R Fau - Muscara, M. Muscara Mn Fau - Malcangio, S.D. Malcang io M Fau - Brain, and S.D. Brain, Hydrogen peroxide is a novel mediator of inflammatory hyperalgesia, acting via transient receptor potential vanilloid 1-de pendent and independent mechanisms. Pain, 2009, 141,135-142,https://doi.org/10. 1016/j.pain.2008.10.025

60. Valério, D.A., S.R. Georgetti, D.A. Magro, R. Casagrande, T.M. Cunha, F.T.M. C. Vicentini, S.M. Vieira, M.J.V. Fonseca, S.H. Ferreira, F.Q. Cunha, and W.A. Verri, Quercetin Reduces Inflammatory Pain: Inhibition of Oxidative Stress an d Cytokine Production. Journal of Natural Products, 2009, 72,1975-1979, https:// doi.org/10.1021/np900259y

61. Kiasalari, Z., T. Rahmani, N. Mahmoudi, T. Baluchnejadmojarad, and M. Rogh ani, Diosgenin ameliorates development of neuropathic pain in diabetic rats: In volvement of oxidative stress and inflammation. Biomedicine \& Pharmacotherap y, 2017, 86,654-661,https://doi.org/10.1016/j.biopha.2016.12.068

62. Ribeiro, R.A., S.M. Vale Ml Fau - Thomazzi, A.B. Thomazzi Sm Fau - Pasch oalato, S. Paschoalato Ab Fau - Poole, S.H. Poole S Fau - Ferreira, F.Q. Ferr eira Sh Fau - Cunha, and F.Q. Cunha, Involvement of resident macrophages an $d$ mast cells in the writhing nociceptive response induced by zymosan and acet ic acid in mice. European Journal of Pharmacology, 2000, 387,111-118,https://d oi.org/10.1016/s0014-2999(99)00790-6

63. Lopez-Luna, J., Q. Al-Jubouri, W. Al-Nuaimy, and L.U. Sneddon, Impact of st ress, fear and anxiety on the nociceptive responses of larval zebrafish. PLoS One, 2017, 12,e0181010,10.1371/journal.pone.0181010 5540279

64. Maximino, C., Modulation of nociceptive-like behavior in zebrafish (Danio reri o) by environmental stressors. Psychology \& Neuroscience, 2011, 4,149-155, htt ps://doi.org/10.3922/j.psns.2011.1.017

65. Schroeder, P.G. and L.U. Sneddon, Exploring the efficacy of immersion analges ics in zebrafish using an integrative approach. Applied Animal Behaviour Scie nce, 2017, 187,93-102,https://doi.org/10.1016/j.applanim.2016.12.003 